\title{
Representatividade LGBT+ na Educação Médica e Covid-19: Construindo Redes de Cuidado e Solidariedade
}

\author{
LGBT+ Equal Representativity in Medical Training and Covid-19: Building Care and Solidarity Networks
}

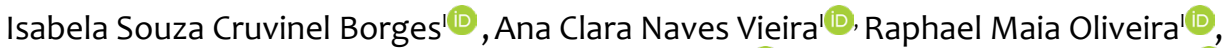
Gabriel Marinho e Silva'(1), Gustavo Antonio Raimondi'

\section{RESUMO}

Introdução: Historicamente, a comunidade LGBT+ enfrenta obstáculos estruturais que interferem na garantia da cidadania plena. Com a pandemia da Covid-19, observa-se que a vulnerabilidade individual da população LGBT+ tornou-se ainda mais intensa e explícita. Diante disso, o presente relato de experiência apresenta e analisa criticamente uma ação de estudantes de Medicina que buscaram promover a representatividade e uma rede de cuidado/apoio e solidariedade entre os(as) estudantes LGBT+ no contexto da pandemia.

Relato de experiência: Este relato descreve a construção de um vídeo "Evoluiu Challenge" por acadêmicos(as) e médicos(as) graduados(as) do curso de Medicina de uma universidade pública brasileira. Diante do isolamento social e da necessidade de representatividade, encorajamento e empoderamento LGBT+ dentro do espaço acadêmico da Medicina, identificou-se a necessidade de ações, como a produção do vídeo, para que os(as) acadêmicos(as) mais vulnerabilizados(as) possam localizar em seus/suas colegas de curso uma rede de apoio, solidariedade e empoderamento. O projeto foi desenvolvido com 20 estudantes e três egressos(as) da mesma instituição. Ao todo, foram mais de 85 mil visualizações. Discussão: Diante da experiência relatada, destaca-se a importância dos avanços tecnológicos como promotores da "aproximação" em tempos de isolamento e distanciamento social. Nesse sentido, o vídeo resultou em significativa visibilidade à população LGBT+ presente no meio médico. Ademais, propiciou representatividade e a construção de uma rede de apoio estudantil, como descrito nas DCN, com a promoção do cuidado.

Conclusão: A partir deste relato de experiência, fica visível a necessidade de implementação de estratégias de ações de apoio para a população LGBT+ nas universidades, como as redes de cuidado/solidariedade. Dessa maneira, assim como o vídeo, essas estratégias potencializarão a construção de um espaço de ensino-aprendizagem mais inclusivo e que seja um oásis do pensamento contra a opressão e espaço de contestação.

Palavras-chave: Minorias Sexuais e de Gênero; Pandemia; Educação Médica; Mídias Sociais; Promoção da Saúde.

\begin{abstract}
Introduction: Members of the LGBT+ community have historically faced structural obstacles denying them the protections and rights guaranteed by full citizenship. The COVID-19 pandemic has caused the vulnerability of these individuals to become even more intense and explicit. In light of these developments, this experience report presents and critically analyzes an action executed by medical students to promote equal representation and a care/support and solidarity network among LGBT+ students during the pandemic.

Experience Report: This report describes the creation of a video entitled "Evoluiu Challenge" by medical students and graduates from a Brazilian public university. In the face of social isolation and the need for LGBT+ equal representation, encouragement and empowerment in medical schools, actions, such as producing the video, were identified as necessary in order to help the most vulnerable academics find a network of support, solidarity and empowerment among their peers. The project was developed with 20 students and 3 graduates from the same institution, with the video being viewed more than 85,000 times. Discussion: The experience reported here highlights the importance of technological advances to promote "togetherness" in times of isolation and social distancing. In this regard, the video increased the visibility of the LGBT+ population in the medical sphere. Furthermore, it fostered equal representation and the construction of a student support network promoting care, as described in the National Curriculum Guidelines.
\end{abstract}

Conclusion: This experience report clearly demonstrates the need to implement strategies for actions that support the LGBT+ community at universities, such as care/solidarity networks. Such strategies, like the video, will therefore leverage the construction of a more inclusive teaching-learning space, representing an oasis of thought against oppression and space for contestation.

Keywords: Sexual and Gender Minorities; Pandemic; Medical Education; Social Media; Health Promotion.

'Universidade Federal de Uberlândia, Uberlândia, Minas Gerais, Brasil.

Correspondência:

Isabela Souza Cruvinel Borges.

Avenida Nicodemos Alves dos Santos, 100, ap. 903, bloco B, Saraiva, Uberlândia, MG, Brasil. CEP: 38408-032.

E-mail: isabelascborges@gmail.com

Recebido em 05/08/20; Aceito em 14/08/20. 


\section{INTRODUÇÃO}

Historicamente, a comunidade de lésbicas, gays, bissexuais, travestis, transexuais e outras identidades de gênero e orientações sexuais (LGBT+) enfrenta obstáculos estruturais que interferem na garantia da cidadania plena, marcada pela liberdade, paz e justiça social. No Brasil, apesar dos avanços no âmbito dos direitos humanos, comportamentos discriminatórios e excludentes, frequentemente naturalizados, caracterizam uma LGBT+fobia estrutural, que persiste no cotidiano desse grupo e o marginaliza constantemente'.

Dentro e fora de casa, a população LGBT+ deparase constantemente com a sensação de medo, reafirmando a posição de vulnerabilidade social, que está associada à fragilização da cidadania, destacando-se pela sujeição à violência e pela desigualdade perante a lei e garantia dos direitos ${ }^{2}$. Nesse contexto, a família consiste em um componente importante da rede de apoio social da população LGBT+³, caracterizada pelo surgimento de vínculos significativos de proteção e suporte, que pode aumentar a resiliência e diminuir os impactos da LGBT+fobia. Contudo, quando ela lida de forma negativa com a aceitação do indivíduo em questão, potencializa a vulnerabilidade já existente ${ }^{4}$.

Diante desse cenário, observa-se que, em muitas situações, essa população é submetida ao "armário"/ silenciamento, em algum nível, nos espaços de socialização. Assim, "sair do armário" torna-se duplamente árduo, uma vez que, embora tenham se assumido, as pessoas LGBT+ presenciam a "necessidade" de manter-se, em algum grau, no "armário", nas variadas situações, a fim de evitar opressão e intolerância ${ }^{5}$. Esses silêncios contribuem para a perpetuação da LGBT+fobia na medida em que mantêm a invisibilidade social e, consequentemente, o discurso dominante branco-cisheteronormativo ${ }^{6}$.

Nesse sentido, no âmbito internacional, a Agenda 2030 da Organização das Nações Unidas (ONU) preconiza a promoção e a proteção dos direitos humanos para a população LGBT+, visando à equidade e à inclusão social, bem como ao cuidado relacionado à saúde7. No Brasil, o Programa Nacional de Direitos Humanos, especificamente a terceira versão ( $\mathrm{PNDH}-3)$, orienta uma articulação entre as instituições sociais, Estado, família e sociedade, visando garantir a livre orientação sexual e identidade de gênero mediante ações programáticas que favoreçam o aumento da visibilidade e o reconhecimento social ${ }^{8}$.

Em face dessa realidade, as Diretrizes Curriculares Nacionais do Curso de Graduação em Medicina(DCN) abrangem áreas referentes à atenção, à gestão e à educação em saúde, de modo a estimular que os(as) estudantes da escola médica sejam aptos(as) a reconhecer as dimensões da diversidade humana, como gênero, sexualidade, raça, etnicidade, entre outras, e exercer o cuidado pautado na universalidade e na equidade, que são princípios e diretrizes do Sistema Único de Saúde (SUS) ${ }^{9,10}$. Ademais, destaca-se que a Medicina é uma instituição composta por indivíduos que, historicamente, participam de formulações de normas, que por vezes incluem e por vezes excluem comportamentos, sujeitos e corpos. Assim, observa-se que, apesar de os diferentes cursos universitários apresentarem diversidade, ainda evidenciam barreiras decorrentes da branco-cis-heteronormatividade ${ }^{11}$. Logo, isso impede que esses indivíduos vivenciem plenamente os direitos humanos, daí a necessidade do desenvolvimento de ações que busquem mitigar esse cenário atual de violências.

Com a pandemia da coronavirus disease 2019 (COVID-19), preconizou-se o distanciamento social ${ }^{12}$, um procedimento que deve ser adotado igualmente por todos(as), porém a maneira como ele atinge cada um(a) é diferente, variando de acordo com o gênero, a raça, a orientação sexual, o poderio econômico e outros ${ }^{13,14}$. Dessa forma, a vulnerabilidade individual da população LGBT+ tornou-se ainda mais intensa e explícita, por causa da ansiedade e do estresse gerados ${ }^{15}$, particularmente em universitários(as), pela falta das atividades habituais e da interatividade social às quais estavam acostumados(as) ${ }^{16}$.

Somado a isso, essa vulnerabilidade também se relaciona ao maior convívio intrafamiliar, o que gera desconfortos psicológicos quando não há aceitação do gênero ou orientação sexual e/ou quando há dificuldades em assumi-los ${ }^{4}$. Afinal, apesar de a família ser considerada uma instituição de maior apoio em outras questões de minorias, frequentemente consiste no ambiente de maior LGBT+fobia ${ }^{6}$. Ademais, nota-se que a redução do convívio dos(as) estudantes com seus círculos afetivos de amizade diminui o apoio diário que possuíam no enfrentamento rotineiro das discriminações sofridas ${ }^{17}$.

Diante disso, o presente relato de experiência apresenta e analisa criticamente uma ação de estudantes de Medicina que buscaram promover a representatividade, caracterizada por uma afirmação de identidade dos(as) LGBT+ que compõem esse meio, no Dia do Orgulho LGBT+, 28 de junho, por meio do uso das mídias e redes sociais, as quais permitiram e possibilitaram a interação entre as pessoas, de modo a promover uma rede de cuidado/apoio e solidariedade entre os(as) estudantes ${ }^{3}$ e, consequentemente, reduzir os efeitos deletérios do distanciamento e isolamento social durante esse período ${ }^{18}$.

\section{RELATO DE EXPERIÊNCIA}

Este relato descreve a construção de um vídeo "Evoluiu Challenge" (Figura 1) por acadêmicos(as) e médicos(as) 
graduados(as) do curso de Medicina da Universidade Federal de Uberlândia (UFU) para celebrar o Mês do Orgulho LGBT+, contextualizando as experiências compartilhadas ao longo do processo, que permitiram a sua produção, e os resultados/ impactos em curto prazo dessa ação. O "Evoluiu Challenge" é uma modalidade de vídeo fundamentada na gravação de pequenos vídeos em que o(a) protagonista realiza movimentos com as mãos ou objetos a fim de produzir a impressão de que houve grandes mudanças em seu visual ${ }^{19}$. O projeto foi idealizado e coordenado por um estudante homossexual do curso após se sentir inspirado em um material de natureza semelhante que circulava nas redes sociais e por identificar a necessidade de representatividade, encorajamento e empoderamento LGBT+ dentro do espaço acadêmico da Medicina.

$\mathrm{O}$ isolamento social imposto pelo atual contexto pandêmico colabora para o agravamento de diversas vulnerabilidades, como as relacionadas à orientação sexual e à identidade de gênero ${ }^{20}$. Esse fato também foi encarado como um pretexto plausível para a confecção do vídeo, uma vez que ele permitiria que os(as) acadêmicos(as) mais vulnerabilizados(as) localizassem em seus/suas colegas de curso uma rede de cuidado/apoio, solidariedade e empoderamento, sendo este último conceito caracterizado pela autodeterminação de sujeitos que buscam uma atuação representativa e concreta de forma democrática e equitativa para conduzir de forma autônoma a própria vida²1.

O projeto foi desenvolvido com 20 estudantes e três egressos(as) da mesma instituição. Todos(as) receberam os convites para participar pelo aplicativo de mensagens WhatsApp ${ }^{\circledR}$. Os pré-requisitos necessários para a participação foram: possuir mais de 18 anos, ser assumidamente LGBT+, consentir com a divulgação de sua imagem nas redes sociais e ser um(a) atual discente, aluno(a) egresso(a) e/ou docente/ preceptor(a) do curso de Medicina da instituição.

A partir de então, criou-se um grupo na plataforma de mensagens WhatsApp ${ }^{\circledR}$, e todos os 23 membros(as) dessa iniciativa foram incluídos(as). Essa estratégia de trabalho em um aplicativo de mensagem permitiu o desenvolvimento de habilidades de trabalho em equipe, como a comunicação de forma afetuosa, o manejo de divergências, o feedback valorativo e a prática do não saber associada à construção compartilhada de conhecimentos ${ }^{22}$. O ambiente de cuidado/ apoio e encorajamento criado dentro do grupo potencializou também a expressão da negritude de um dos discentes, que, ao gravar seu vídeo, portava um cartaz escrito: "futuro médico + preto + LGBT = ORGULHO".

A primeira etapa do projeto foi a divulgação do vídeo que inspirou o seu desenvolvimento e a explicação da importância e do impacto que esse material poderia produzir não apenas na
Figura 1. Capa de um vídeo "Evoluiu Challenge" produzido pelos(as) acadêmicos(as) e egressos(as) LGBT+ do curso de Medicina da UFU, com o título LGBT+ Pride Month (Mês do Orgulho LGBT+)

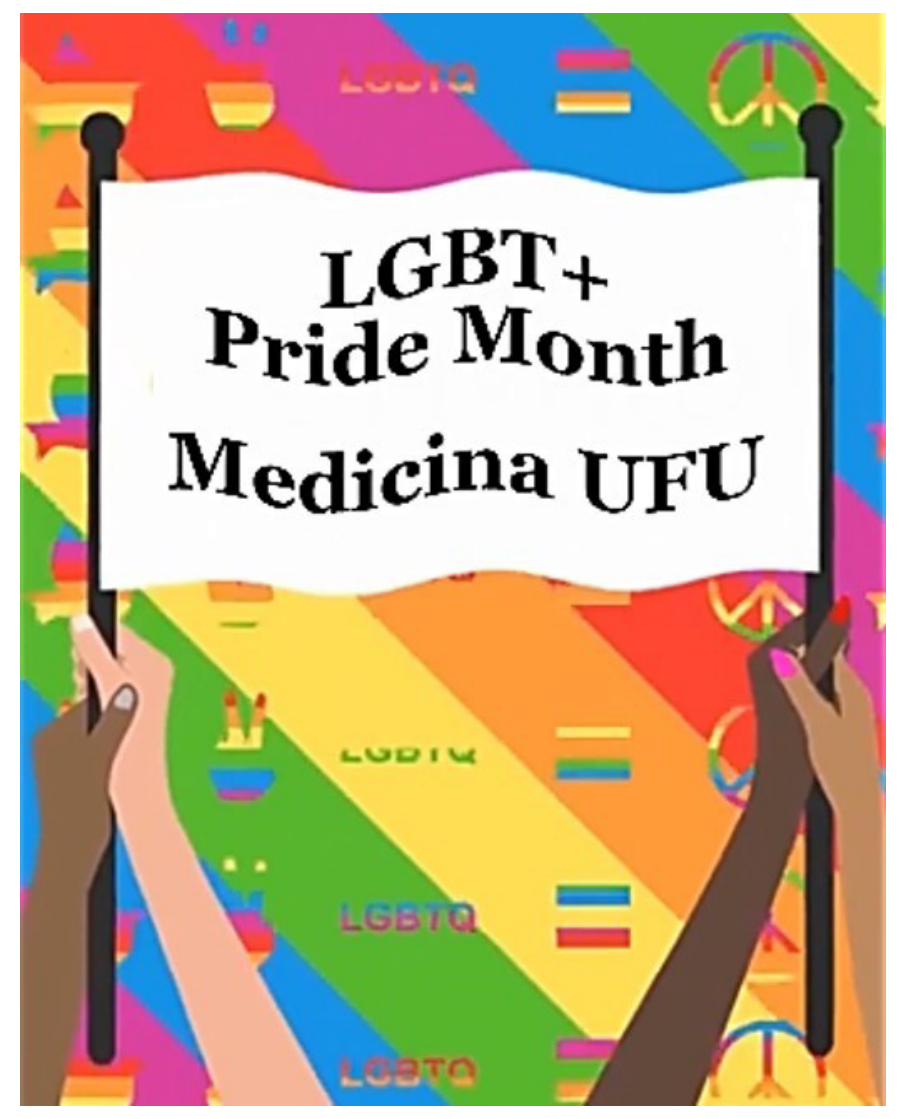

Fonte: Elaborada pelos autores(as).

comunidade acadêmica de Medicina, mas também nos cursos da área da saúde como um todo. Entre os impactos almejados, destacam-se os seguintes: a importância de discutir e reconhecer a existência da diversidade de gênero e sexual, a relevância da representatividade em ambientes acadêmicos tidos como conservadores, a demonstração da existência de populações vulnerabilizadas no curso de Medicina e a imprescindibilidade de essa população se configurar em uma rede de cuidado/apoio mútuo, especialmente no contexto da Covid-19.

O próximo passo do projeto foi o repasse de orientações para a confecção dos vídeos. Cada integrante do grupo deveria gravar dois vídeos diferentes. No primeiro, a pessoa iria aparecer caracterizada como um(a) profissional da área da saúde, simulando estar recebendo de um(a) outro(a) integrante um objeto relacionado ao curso de Medicina. Ao finalizar esse primeiro vídeo, cada pessoa deveria cobrir a lente da câmera utilizada na gravação. No segundo vídeo, a mesma pessoa deveria aparecer destampando a lente da câmera, trajando algum elemento ou efeito de vídeo que o(a) caracterizasse como membro(a) da comunidade LGBT+ (por exemplo, camiseta com as cores da bandeira LGBT+) e, ao final, simular 
estar passando para o(a) próximo(a) participante algum objeto que também o(a) relacionasse ao curso de Medicina (por exemplo, estetoscópio). Essas orientações foram dispostas dessa forma para construir a impressão de continuidade e mudança repentina, características dos vídeos que seguem o modelo "Evoluiu Challenge".

O terceiro passo do projeto foi realizar a junção dos vídeos de cada um(a) dos(as) participantes, de forma a tornar o vídeo um só. A música escolhida como pano de fundo foi "Born this way"23, da cantora Lady Gaga, por se tratar de um hino de exaltação às diferenças e às diversidades ${ }^{24}$. Ademais, a canção selecionada possui um trecho que se correlaciona com o programa "Nascidos Livres e Iguais" da ONU, que tem como propósito estabelecer os deveres fundamentais que os Estados possuem para com a população LGBT+ ${ }^{25}$.

A última etapa do projeto foi a divulgação na rede social Instagram ${ }^{\circledR}$. Cada integrante publicou o vídeo em sua conta particular. Quatro participantes, embora assumidamente LGBT+, tomaram a decisão de não publicar o vídeo para evitar desavenças familiares. A divulgação do vídeo também contou com o apoio de outras contas da plataforma, como associação atlética acadêmica, diretório acadêmico, digital influencers, entre outros. $O$ vídeo teve uma resposta positiva que surpreendeu a todos(as). Ao todo, foram mais de 85 mil visualizações, com vários comentários positivos. Por intermédio do apoio recebido nas redes sociais, alcançaramse diversos estados da União, como Rondônia, que tem uma distância geográfica significativa em relação ao estado de Minas Gerais e que foi mencionado de forma específica em um comentário muito positivo de uma internauta. Estudantes LGBT+ da área da saúde de outras universidades também entraram em contato dizendo sentir-se representados(as) e encorajados(as) a produzir material semelhante.

Este relato de experiência foi construído pelo discente idealizador do projeto, três discentes participantes e um docente orientador. Portanto, enquadra-se no item VIII do artigo $1^{\circ}$ da Resolução $n^{\circ}$ 510, de 7 de abril de 2016, do Conselho Nacional de Saúde, não necessitando, assim, de submissão ao Comitê de Ética em Pesquisa com Seres Humanos ${ }^{26}$.

\section{DISCUSSÃO}

Diante da experiência relatada, destaca-se a importância dos avanços tecnológicos como promotores da "aproximação" em tempos de isolamento e distanciamento social, visto que as redes sociais e plataformas on-line permitiram a proximidade virtual e a inovação na forma de se expressar ${ }^{18}$. Nesse sentido, o vídeo resultou em uma significativa visibilidade à população $\mathrm{LGBT}+$ presente no meio médico, muitas vezes conservador e branco-cis-heteronormativo ${ }^{11}$, atingindo uma escala ainda maior que a academia local. Ademais, propiciou representatividade, principalmente nesse contexto pandêmico e de avanço do conservadorismo, que reiteram a violência estrutural de/em nossa sociedade'.

Essa representatividade, decorrente do alcance obtido, gerou aumento da responsabilidade social e do compromisso dos(as) estudantes de Medicina da UFU como cidadãos(ãs) e profissionais da saúde que buscam promover os direitos humanos. Ao tomar conhecimento dos desafios da sociedade contemporânea, o indivíduo torna-se responsável por essa realidade, ainda que externa a ele(a) ${ }^{27}$.

Diante dessa perspectiva e considerando que a universidade é uma instituição pública pautada na formação de cidadãos(ãs)-profissionais, nota-se que os(as) estudantes reconheceram a importância de buscar não só a promoção de saúde, como também a justiça social. Desse modo, o vídeo contribuiu para a construção de uma sociedade democrática, marcada pelo respeito à cidadania pública ${ }^{28,29}$.

Sob outro panorama, referente à tecnologia, destacase sua importância para a formação de uma rede de cuidado/ apoio estudantil, como descrito nas DCN3,9, com a promoção do cuidado, inicialmente do autocuidado, visto que o grupo criado no WhatsApp ${ }^{\circledR}$ foi mantido após a organização do vídeo, visando perpetuar o clima de união e solidariedade surgido. Houve ampla troca de experiências sobre aceitação e preconceito, contando com relatos tanto de egressos(as) como de estudantes dos períodos iniciais da graduação. Ressaltase a diferença geracional nos comentários: enquanto os(as) graduados(as) falaram sobre a impossibilidade de realizar um vídeo como esse em sua época de faculdade, tanto pelo árduo e intenso preconceito como pela menor quantidade de pessoas assumidamente LGBT+, os(as) acadêmicos(as) citaram a ótima receptividade que tiveram da maioria dos(as) colegas de curso, apesar de presenciarem algumas situações de LGBT+fobia.

Diante disso, existem estratégias para fornecer apoio à comunidade LGBT+ nas universidades, como ocorre com o mentoring. O programa de mentoring, presente, por exemplo, no curso de Medicina da Universidade de São Paulo (USP), promove uma troca de saberes entre estudantes de diversos períodos da graduação, por meio de encontros periódicos ${ }^{30}$. Ele gera um espaço seguro e acolhedor, contribuindo não somente para a formação pessoal e profissional dos(as) estudantes, como também para a promoção de saúde deles(as). Nos Estados Unidos, algumas universidades propõem um mentoring para grupos específicos, visando atender às necessidades de indivíduos sub-representados e sujeitos a algum grau de vulnerabilidade ${ }^{31}$. Nesse contexto, seria de extrema relevância a criação de um projeto semelhante, voltado aos(às) estudantes LGBT+, nas faculdades de Medicina de todo o Brasil. 
Aliado a isso, é essencial que as universidades implementem políticas próprias que visem garantir o exercício pleno da cidadania e da dignidade desses indivíduos, a exemplo da Política de Diversidade Sexual e de Gênero da UFU. Essa política objetiva combater a discriminação e promover equidade de direitos e de oportunidades, por meio de: ações afirmativas; serviços contra violência; incentivo a pesquisas, projetos de extensão e eventos sobre diversidade sexual; introdução de conteúdos sobre essa questão na grade curricular; e publicações institucionais e campanhas a fim de promover conscientização e dar visibilidade à população LGBT+32.

Apesar de a invisibilidade de décadas passadas ter sido gradualmente "amenizada", o empoderamento e a maior visibilidade dos dias atuais explicitaram o processo de construção de estereótipos. Pode-se citar, por exemplo, a imagem de "gay afeminado" que as novelas brasileiras constantemente trazem ${ }^{33}$. No contexto da Medicina, esses estereótipos também se manifestam diariamente: conforme comentado pelos(as) participantes no grupo, esse paradigma mostra-se frequentemente relacionado às especialidades médicas, sendo um dos exemplos citados o preconceito existente diante de homens homossexuais atuando em áreas cirúrgicas, principalmente na ortopedia e traumatologia. O problema dos estereótipos é que eles são incompletos e fazem da história relacionada a esse estereótipo a única passível de ser contada e/ou lembrada e/ou possível de ser vivida $^{34}$. Com a presença dos(as) 23 participantes do vídeo, cada apresentação de si proporcionava uma problematização desses estereótipos e buscava ampliar as possibilidades para além de "uma história única".

Paralelamente a isso, é perceptível também a interseccionalidade, caracterizada como uma ferramenta que leva em consideração mais de uma forma de opressão simultaneamente, por causa da interação de vários marcadores sociais, como gênero, sexualidade, raça, etnicidade, renda, entre outros ${ }^{35}$. Diante disso, 30\% dos(as) participantes do vídeo que se autodeclaram negros(as) ou pardos(as) explicitam a dupla opressão que podem vivenciar: a primeira ligada ao racismo estrutural e institucional, e a segunda relacionada à aceitação de sua sexualidade, o que os(as) impede novamente de ser acolhidos(as) e integrados(as), gerando uma intensa solidão afetiva ${ }^{36}$.

Nesse sentido, a frase "futuro médico + preto + LGBT = ORGULHO”, presente no vídeo, explicita a importância de pensar a educação médica em relação ao racismo estrutural, a violência contra a população negra e a relação da branquitude em uma cultura/sistema racista. Com isso, fica evidente a relevância das questões étnico-raciais na formação profissional, que, em diálogo com a Política Nacional de Atenção Integral à População Negra e as outras políticas da equidade em saúde, promovem um cuidado em saúde equânime, antirracista, humanista e ético ${ }^{37}$.

Sobre a vulnerabilidade, ressalta-se a existência de grande discrepância dentro do próprio grupo LGBT+, em relação tanto à visibilidade e à aceitação, quanto à ocupação de espaços pelos indivíduos. As travestis e os(as) transexuais são ainda mais afetados(as) por essa violência estrutural, fato que está diretamente ligado ao sistema binário, brancocisnormativo, higienista e transfóbico das instituições ${ }^{6}$. Mesmo diante do discurso das instituições em defesa da diversidade social $^{38}$, podem-se ampliar as ações para a promoção da equidade, tendo em vista que cada grupo possui particularidades e necessidades específicas que precisam ser conhecidas e atendidas.

Nesse contexto, nota-se a primeira limitação do vídeo: a ausência de estudante(s) representando a letra "T", já que não se obteve conhecimento, durante o recrutamento das pessoas para o vídeo, de discentes, docentes ou técnico(as)administrativos(as) transexuais ou travestis. Além disso, não se teve informação sobre a existência de estudantes LGBT+ indígenas, o que é uma outra limitação do vídeo e que corresponde, também, à baixa representatividade dessa população na universidade ${ }^{39}$.

Ademais, explicita-se outra limitação para uma maior participação de pessoas nessa proposta: a dificuldade de assumir-se, quanto à orientação sexual ou à identidade de gênero. O processo de descoberta do próprio ser é árduo e muitas vezes confuso. É a partir das próprias experiências que o indivíduo se conhece e forma sua identidade. Em discordância com esse fluxo livre de acontecimentos, a sociedade impõe regras contraditórias sobre público e privado, sigilo e exposição 5 .

Nesse cenário de tamanho preconceito, binaridade e dicotomia, é comum que as pessoas encontrem dificuldades para se assumir, seja para outros, seja para si próprias. Assim, tem-se que os 23 indivíduos presentes no vídeo representam uma pequena parcela de todas as pessoas desse grupo que estão cursando ou cursaram Medicina na UFU. Isso mostra que, mesmo diante de todas as adversidades, essas pessoas existem, (sobre)vivem e estão presentes nesses e em todos os outros espaços.

\section{CONCLUSÃO}

A pandemia e, consequentemente, o isolamento social tiveram impactos na vida das pessoas, intensificando situações de vulnerabilidade, como na população LGBT+. Diante disso, a produção do vídeo obteve resultados positivos e alcance nacional por meio da promoção da diversidade. Assim, concluiu-se que o exercício constante da representatividade contribui para o rompimento dos estereótipos que foram 
criados para os(as) LGBT+ como sua única identidade imutável. Ademais, corrobora a necessidade de desconstruir o padrão branco-cis-heteronormativo sob o qual o ambiente médico foi erigido, além de proporcionar uma rede de apoio para os(as) que ocuparam, ocupam e ocuparão esse espaço.

Sendo a universidade um espaço público por excelência, lugar legitimado de fala, de produção de conhecimento e de busca de transformação, haveria de se supor a inexistência da LGBT+fobia em vez de sua reprodução, no entanto ainda é presente essa problemática. Assim, fica visível a necessidade de implementação de estratégias de ações de apoio para a população LGBT+ nas universidades, como as redes de cuidado/ apoio e solidariedade e o mentoring. Dessa maneira, assim como o vídeo, potencializarão a construção de um espaço de ensino-aprendizagem mais inclusivo e que seja um oásis do pensamento contra a opressão e espaço de contestação. Entretanto, é válido lembrar que esse papel não é apenas das instituições de ensino ou dos órgãos estatais, mas também de todos(as) os(as) cidadãos(ãs), que devem sempre ser promotores(as) dos direitos humanos.

\section{AGRADECIMENTOS}

Agradecemos a todos(as) os(as) discentes e egressos(as) LGBT+ do curso de Medicina da UFU que participaram da construção do vídeo e da rede de apoio edificada a partir dessa experiência. Gostaríamos de agradecer também aos(às) idealizadores(as) e participantes do vídeo que serviu de inspiração para a construção do nosso. Os agradecimentos também se estendem a todos(as) aqueles(as) que contribuíram com a divulgação do material em suas redes sociais, possibilitando, dessa forma, umamploalcance e número de visualizações. Também somos gratos(as) a todos(as) que apreciaram nosso trabalho e se sentiram inspirados(as) por ele. De igual maneira, agradecemos a todos(as) aqueles(as) que, de alguma forma, apoiam, incentivam e promovem redes de apoio e solidariedade criadas ao longo dos processos formativos e de cuidado em saúde. Por último, mas não menos importante, gostaríamos de agradecer de forma especial ao médico Vinícius Borges, dono do perfil @doutormaravilha, e à médica Marcela Mc Gowan, que foram as pessoas por meio das quais tivemos acesso ao vídeo inspirador do nosso projeto e que contribuíram de forma incrível com a divulgação do nosso material.

\section{CONTRIBUIÇÃO DOS AUTORES}

Todos(as) os(as) autores(as) participaram ativamente de todas as etapas de elaboração do manuscrito.

\section{CONFLITO DE INTERESSES}

Os(as) autores(as) declaram não haver conflito de interesses neste estudo.

\section{REFERÊNCIAS}

1. Pedra CB. Direitos LGBT: a LGBTfobia estrutural na arena jurídica [dissertação]. Belo Horizonte: Universidade Federal de Minas Gerais; 2018.

2. Sevalho G. O conceito de vulnerabilidade e a educação em saúde fundamentada em Paulo Freire. Interface Comun Saúde Educ. 2018;22(64):177-88.

3. Juliano MCC, Yunes MAM. Reflexões sobre rede de apoio social como mecanismo de proteção e promoção de resiliência. Ambient Soc. 2014;17(3):135-54.

4. Braga IF, Oliveira WA de, Silva JL da, Mello FCM de, Silva MAI. Violência familiar contra adolescentes e jovens gays e lésbicas: um estudo qualitativo. Rev Bras Enferm. 2018;71(3):1295-303.

5. Sedgwick EK. A epistemologia do armário. Cad Pagu. 2007;(28):19-54.

6. Silva LV da, Barbosa BRSN. Sobrevivência no armário: dores do silêncio LGBT em uma sociedade de religiosidade heteronormativa. Rev Estudos de Religião. 2016;30(3):129-54.

7. Organização das Nações Unidas. Agenda 2030. In: ONU. Agenda 2030 for LGBTI Health and Well-being. Brasília: ONU; 2017. p. 1-48 [acesso em 20 jul 2020] Disponível em: https://nacoesunidas.org/pos2015/agenda2030/.

8. Brasil. Programa Nacional de Direitos Humanos (PNDH-3). Brasília: Secretaria Especial de Direitos Humanos da Presidência da República (SEDH/PR); 2009.

9. Brasil. Ministério da Educação. Conselho Nacional de Educação. Câmara de Educação Superior. Resolução $n^{\circ}$ 3, de 20 de junho de 2014. Institui Diretrizes Curriculares Nacionais do Curso de Graduação em Medicina e dá outras providências. Diário Oficial da União, Brasília; 23 jun. 2014; Seção 1, p. 8-11.

10. Brasil. Ministério da Saúde. Secretaria Executiva Sistema Único de Saúde (SUS): princípios e conquistas. Ministério da Saúde, Secretaria Executiva - Brasília: Ministério Saúde; 2020.

11. Raimondi GA, Teixeira FB, Moreira C, Barros NF. Corpos (não) controlados: efeitos dos discursos sobre sexualidades em uma Escola Médica Brasileira. Rev Bras Educ Med. 2019; 43(3):16-26.

12. Islam N, Sharp SJ, Chowell G, Shabnam S, Kawachi I, Lacey B, et al Physical distancing interventions and incidence of coronavirus disease 2019: natural experiment in 149 countries. The BMJ. 2020;370:m2743.

13. Sun J, He W, Wang L, Lai A, Ji X, Zhai X, et al. Covid-19: epidemiology, evolution, and cross-disciplinary perspectives. Trends Mol Med. 2020;26(5):483-95.

14. Silver RC. Surviving the trauma of Covid-19. Science. 2020;369(6499):11.

15. Gomes R, Murta D, Facchini R, Meneghel SN. Gênero, direitos sexuais e suas implicações na saúde. Ciênc Saúde Colet. 2018;23(6):1997-2006.

16. Maia BR, Dias PC. Ansiedade, depressão e estresse em estudantes universitários: o impacto da Covid-19. Estud Psicol (Campinas). 2020;37:e200067.

17. Davi EHD. Resistências e recusas: a cultura LGBT contrapondo-se a homofobia em Uberlândia. Cad Esp Fem. 2011;24(1):141-61.

18. Krogan N, Vousden K, Doudna J, Schulman BA, Gingras A-C, Dikic I. Science in the wake of the pandemic: how will Covid-19 change the way we do research? Mol Cell. 2020;79(1):9-10.

19. Cardoso B. O que é Evoluiu Challenge? Entenda o vídeo que virou moda no Instagram. TechTudo; 2019 [acesso em 28 jul 2020]. Disponível em: https:// www.techtudo.com.br/noticias/2019/09/o-que-e-evoluiu-challengeentenda-o-video-que-virou-moda-no-instagram.ghtml.

20. Carman M, Bourne A, Fairchild J. Covid-19: impacts for LGBTIQ communities and implications for services: a research briefing paper by Rainbow Health Victoria. Melbourne: Rainbow Health Victoria, Australian Research Centre in Sex, Health and Society, La Trobe University; 2020. 
21. Kleba ME, Wendausen A. Empoderamento: processo de fortalecimento dos sujeitos nos espaços de participação social e democratização política. Saúde Soc. 2009;18(4):733-43.

22. Dias LF, Vargas LG, Silva GM e, Souza TG de, Santos CAG, Raimondi $\mathrm{GA}$, et al. Promoção da saúde: coerência nas estratégias de ensinoaprendizagem. Rev Bras Educ Med. 2019;43(1):641-51.

23. Germanotta S, Laursen J. Born this way. New York City: Interscope Records; 2011 [acesso em 25 jul 2020]. Disponível em: https://www. youtube.com/watch?v=fn8JP4rUP2l.

24. Henn RC, Gonzatti C. Don't be a drag, just be a queer: Lady Gaga e semiodiversidade em redes digitais do jornalismo de cultura pop. Contracampo. 2019;38(1):35-50.

25. Pillay N. Nascidos livres e iguais: orientação sexual e identidade de gênero no regime internacional de direitos humanos. Brasília: Unaids Brasil, 2013.

26. Brasil. Conselho Nacional de Saúde. Resolução $n^{\circ} 510$, de 7 de abril de 2016. Dispõe sobre as normas aplicáveis a pesquisas em Ciências Humanas e Sociais. Diário Oficial da União, Brasília; 24 maio 2016; Seção 1.

27. Barros CMP. Responsabilidade social universitária: um estudo de caso no curso de Medicina da Universidade Federal do Ceará - campus de Sobral [dissertação]. Fortaleza: Universidade Federal do Ceará, 2009.

28. Oliveira FP de, Santos LMP, Shimizu HE. Responsabilidade social das escolas médicas e representações sociais dos estudantes de Medicina no contexto do Programa Mais Médicos. Rev Bras Educ Med. 2020;43(1):462-72.

29. Sobrinho JD. Universidade fraturada: reflexões sobre conhecimento e responsabilidade social. Avaliação (Campinas). 2015;20(3):581-601.

30. Bellodi PL. O Programa Tutores e a integração dos calouros na FMUSP. Rev Bras Educ Med. 2004;28(3):204-14.
31. Kosoko-Lasaki O, Sonnino RE, Voytko ML. Mentoring for women and underrepresented minority faculty and students: experience at two institutions of higher education. J Natl Med Assoc. 2006;98(9):1449-59.

32. Universidade Federal de Uberlândia. Resolução $n^{\circ} 10$, de 30 de agosto de 2019, do Conselho Universitário. Estabelece a Política de Diversidade Sexual e Gênero da Universidade Federal de Uberlândia. Uberlândia; 2019 [acesso em 28 jul 2020]. Disponível em: http://www.reitoria.ufu.br/ Resolucoes/ataCONSUN-2019-10.pdf.

33. Silva DM da. Diversidade na mídia: a imagem do gay estereotipado [trabalho de conclusão de curso]. Varginha: Centro Universitário do Sul de Minas; 2018.

34. Adichie $\mathrm{CN}$. O perigo de uma história única. São Paulo: Companhia das Letras; 2019.

35. Kyrillos $\mathrm{GM}$. Uma análise crítica sobre os antecedentes da interseccionalidade. Rev Estud Fem. 2020;28(1):e56509.

36. Veiga L. As diásporas da bixa preta: sobre ser negro e gay no Brasil. Tabul Let. 2018; 12(1):77-88.

37. Raimondi GA, Souza LP de, Moraes VHA, Afonso DH, Borret RHES, Tourinho FSV, et al. Posicionamento do Grupo de Trabalho Populações (In) Visibilizadas e Diversidades a respeito das violências contra a população negra e do racismo estrutural. Rev Bras Educ Med. 2020;44(3):e093.

38. Silveira TR da. Reflexões sobre as pessoas LGBT nas organizações públicas: a diversidade sexual no discurso institucional de uma instituição de ensino federal [dissertação]. Vitória: Universidade Federal do Espírito Santo; 2016.

39. Bergamaschi MA, Doebber MB, Brito PO. Estudantes indígenas em universidades brasileiras: um estudo das políticas de acesso e permanência. Rev Bras Estud Pedagog. 2018;99(251):37-53. 